\title{
Editorial comment - volume 60 of Fungal diversity
}

\author{
Special issue dedicated to the COST Action FA1103, \\ "Endophytes in Biotechnology and Agriculture"
}

\author{
Marc Stadler • Kevin D. Hyde \\ Published online: 4 June 2013 \\ (C) Mushroom Research Foundation 2013
}

Summary: The European Cooperation in Science and Technology (COST) aims to promote joint interdisciplinary research in various areas and provides substantial funding to international consortia to conduct networking activities such as workshops, training schools, and short time international student exchanges. Even though primarily European Scientists are eligible to propose COST Actions and to receive funding, the international community can and does participate. This special issue is dedicated to a COST Action FA1103 on biotechnological and agricultural exploitation of endophytes, entertained by 150 scientists from over 20 countries. Eleven original papers, one review and two non COST Action papers have been compiled, all of which are dealing with various aspects of fundamental and applied research on fungal endophytes. The broad spectrum of the contributions, which are representative of the scientific scope of the Action, is illustrated by reports on innovative methods for all taxa inventories (molecular ecology), studies relating to bioprospecting. The utility of the newly arising "-omics" technologies, above all for the functional characterisation of these organisms in view of potential beneficial applications for humankind is thus emphasised. The spectrum of included publications extends from detection and monitoring of these cryptic organisms, their isolation and taxonomic classification in the scope of a One-Fungus-One Name Concept, their exploitation for novel bioactive compounds, to the evaluation of their ecological importance. Exciting new results on the ecology of the NeotyphodiumPoaeceae symbiosis and a success story of their utility in

\section{Stadler}

Department Microbial Drugs, Helmholtz-Zentrum für

Infektionsforschung GmbH, Inhoffenstrasse 7,

38124 Braunschweig, Germany

e-mail: marc.stadler@helmholtz-hzi.de

\section{K. D. Hyde $(\bowtie)$}

Institute of Excellence in Fungal Research, and School of Science,

Mae Fah Luang University, Chiang Rai, Thailand

e-mail:kdhyde3@gmail.com biocontrol are presented. On the other hand, a possible sound explanation is given for the failure to attain sustainable biotechnological production of taxol from cultures of fungal endophytes. Participation in the COST Action FA1103 will broaden the expertise of Early-Stage Researchers, and such funding schemes should eventually be adopted by the global mycological community.

The European Cooperation in Science and Technology (COST) programme aims to establish pan-European research networks on interdisciplinary, topical research themes that are in the scope of the goals of the research framework of the European Commission. COST Actions can be granted after proposals of scientist consortia comprising members from at least five different countries in various domains. Those include, e.g., Biomedicine and Molecular Biosciences (BMBS), Chemistry and Molecular Sciences and Technologies (CMST), Earth System Science and Environmental Management (ESSEM), Food and Agriculture (FA), Forests, their Products and Services (FPS), and Trans-Domain (TD) activities. Successful applications receive substantial funding for networking activities, such as conferences, short time student exchanges, training schools and dissemination activities

Initiated in 2010, the COST Action FA1103, "Endophytes in Biotechnology and Agriculture" has so far been joined by 20 countries, and scientists from over 50 institutions are actively involved in research into the exploitation of endophytic fungi and bacteria in biotechnology and agriculture. The Action is divided into four thematic working groups (WG): WG1 (Ecology of endophytes), WG2 (Identification of new competent endophytes), WG3 (Development of new microbial inocula), and WG4 (New industrial products in life sciences).

\footnotetext{
${ }^{1}$ For more information see: www.endophytes.eu (Action website), and http://www.cost.eu/domains_actions/fa/Actions/FA1103 (corresponding COST website).
} 
The papers included in the current special issue of Fungal Diversity deal with topics of all workgroups except for WG3.

An account of the current and forthcoming activities of the Action has been given in IMA Fungus by Stadler (2013) and regular updates can be found on the corresponding websites (http://www.cost.eu/domains_actions/fa/Actions/FA1103 and http://www.endophytes.eu/). This information is not repeated here. Instead, we have compiled a summary of the contributions included in the current special issue, linking these papers to the major objective of the FA1103 Action: “...identification of bottlenecks limiting the use of endophytes in biotechnology and agriculture and ultimately provide solutions for the economically and ecologically compatible exploitation of these organisms"

Four contributions in this issue deal with systemic, vertically transmitted endophytes and the model NeotyphodiumPoaeceae symbiosis. This phenomenon has been studied intensively and has even resulted in commercial applications. Johnson and co-authors $[1]^{2}$ summarise their keynote lecture of the COST FA1103 workshop (Italy, November 2012) entitled "The exploitation of Epichloae endophytes for agricultural benefit". This review demonstrates how multidisciplinary research can result in innovative strategies to ultimately attain increased pasture performance, utilising fungal endophytes. Two concurrent original research papers by Gundel and coauthors [2,3] also provide case studies relating to the same topic. The first deals with symbiotic interactions as drivers of trade-offs in plants using the example of fungal endophytes on tall fescue (Schedonorus phoenix). In particular, the influence of the endophytes on the relationship between plant biomass and on the trade-off between number and weight of panicles (RPN) is explored. The endophytes seem to affect such tradeoffs in tall fescue plants in a complex manner, and a number of contributing biological and abiotic factors are discussed. The second paper compares the effects of Neotyphodium coenophialum on three European wild populations of tall fescue vs. the forage cultivar "Kentucky-31". It was found that the endophyte increases tall fescue performance in general, but the differences between wild populations and cultivars indicate adaptation to local habitats and agronomic management, respectively. The results also suggest that certain plant genotype-endophyte combinations found within populations result from local selection pressures. Zabalgogeazcoa and coauthors [4] provide a case studies for the non systemic fungal endophytes in Festuca rubra plants infected by Epichloë festucae in subarctic habitats. These case studies impressively reveal how much work remains to be done in both the laboratory and in the field, to reach the goal of providing

\footnotetext{
${ }^{2}$ Numbers in square brakets [1-14] indicate the order of the papers in this issue.
}

sustainable solutions for the economically and ecologically compatible exploitation of fungal endophytes.

Other papers included in this special issue focus more on basic research, especially with respect to the ecology of the endophytes and the elucidation of their life cycle.

Vázquez de Aldana [5] and co-workers analysed the endophytic fungi in surveys conducted in 14 grass species and found that some of the most frequent taxa on each grass were also present across several host grasses. These taxa (Alternaria, Epicoccum, Cladosporium and Fusarium) produce abundant spores, and are commonly encountered in air samples where their spores, which are important respiratory allergens, attain high atmospheric concentrations. The authors emphasise the potential importance of this phenomenon, as an important link between climate, plant biology and public health.

Unterseher and co-authors [6] have studied the level of seasonal overlap of cultivable microfungi in living and decaying tissues of Fagus sylvatica in Germany using dilution-to-extinction cultivation over 3 years. Based on microscopic identification and sequencing ITS DNA, a substantial compositional and phylogenetic overlap between leaf and litter fungi was revealed. The data from cultivated leafinhabiting beech endophytes were compared with a 454 sequence data set from beech phyllosphere, allowing the partition of species lists into active fungal endophytes, fungal "epiphytes" and dormant fungal propagules. Another molecular ecology study by Peršoh [7] investigated factors shaping the endophytic community structure in a hemiparasitic plant, Viscum album ssp. austriacum, and its host Pinus sylvestris, using pyrosequencing of rRNA genes. Fungal operational taxonomic units (154) represented by 953,385 sequences, were found in at least two samples from Viscum album ssp. austriacum and/or its Pinus sylvestris host. In contrast to an earlier, cultivation based assessment (Peršoh et al. 2010), where predominantly xylarialean endophytes had been recovered from the same host-parasite system, the culture-independent approach predominantly yielded zygomycetes of the genus Morteriella. The study also revealed that host and/or organ preferences of putatively saprotrophic fungi are predominantly responsible for compositional differences in the endophytic fungal communities

García and co-authors [8] have attempted to establish the "model plant", Arabidopsis thaliana as model system for an integral approach to studying the principles governing the endophytic lifestyle, taking advantage of the molecular tools and the abundant knowledge accessible from this host plant. They analysed the culturable endophytic mycobiota associated with the model plant and studied the effects of biotic and abiotic factors with regard to the species composition of the endophytic endophyte species composition. 
Wikee and co-workers [9] investigated the distribution of a single endophyte species, Phyllosticta capitalensis. This species has a cosmopolitan distribution occurring on more than 70 plant families as an endophyte, but also as a pathogen. Unlike other pathogenic Phyllosticta species P. capitalensis is easy to isolate and grows relatively fast. Thus in studies where a pathogen is isolated from a host from diseased tissues rather than via single spores, or where Phyllosticta species are isolated for screening purposes, one should expect to isolate this single widespread species. This study has important implications for researchers screening for novel compounds or establishing the causal agents of plant disease.

Pažoutová and co-authors [10] have addressed various aspects of endophyte research (molecular and chemical ecology, bioprospecting, and even taxonomic classification of endophytes in the era of an unified fungal nomenclature) simultaneously: A xylariaceous endophytic species closely associated to the willow wood wasp, Xiphydria prolongata, was characterised by chemical profiling, molecular phylogeny and morphological studies and recognised as new. Notably, the identity of this new species, Daldinia hawksworthii, was only safely established, based on concurrent extensive monographic work by Stadler et al. (2013) that provided sound reference data on several thousands of specimens and cultures of Daldinia and related Xylariaceae. A new, apparently specific bioactive secondary metabolite was also discovered from the new species, and evidence first on the utility of GC-MS profiling for Xylariaceae chemotaxonomy was presented.

A paper submitted during preparation of this issue, although not related to Cost Action is included as it deals with an important issue. Delaye and co-authors [11] investigate the switches of life modes between endophytes and necrotrophic and biotrophic pathogens. They conclude that switches from endophytic to necrotrophic pathogenic lifestyles or vice versa have occurred on several occasions, whereas biotrophy usually represents a derived and evolutionarily stable trait.

Two papers dealing with the utility of endophytes for bioprospecting are also included. It has recently become common practice to focus on the cultivable endophytic mycota of important medicinal plants (Huang et al. 2009; Kusari et al. 2012) and screen them for the occurrence of the plant metabolites. Even though a number of important plant metabolites were already detected in the corresponding endophytes, mostly in trace amounts, the few secondary metabolites from endophytic fungi that have hitherto given rise to sustainable production processes in view of pharmaceutical development (e.g. nodulisporic acid; cf. Bills et al. 2012), all seem to constitute original metabolites from the fungi that have never been isolated from a plant (Aly et al. 2010; Debbab et al. 2011, 2012; Kesting et al. 2011). Nevertheless, medicinal plants have been proven to be a rich source of novel chemical entities (Aly et al. 2011; Maneerat et al. 2012), and further studies will certainly be rewarding.

Kusari and co-authors [12] have undertaken a case study on endophytic fungi from Cannabis sativa, and surprisingly found that the majority of the 30 endophyte strains belonged to the genus Penicillium, which has hitherto been thought to be less well-represented among the endophytic mycota than in other habitats such as soil. Penicillium and other genera represented among the isolated endophyte strains are known to be prolific sources of novel bioactive compounds. Promising antagonistic effects in vitro of the endophytes were observed in dual culture against the Cannabis pathogens, Botrytis cinerea and Trichothecium roseum, and therefore chances are high that novel secondary metabolites with interesting bioactivities can be obtained from an in-depth characterisation of the novel strains.

Tejesvi et al. [13] describe the discovery and bioactivities of a novel antimicrobial peptide from an endophytic strain of Fusarium. The authors used transcriptomics, combined with analytical chemistry and chromatography to isolate and characterise the new compound, which showed moderate, broad spectrum antibiotic activities and has a molecular weight of over $6.000 \mathrm{Da}$. A straightforward method for sustainable production of the novel peptide, named Trtesin, after cloning and heterologous expression was also developed. Interestingly, this innovative class of bioactive metabolites has hitherto been neglected, since conventional bioprospecting approaches have mainly targeted medium polar to lipophilic compounds with molecular weights of $<2,000 \mathrm{Da}$. A systematic screening of endophytic and nonendophytic fungi for such "large antibiotics" will in all likelihood reveal numerous novel chemical entities with potential utility, which can very likely be made more easily accessible by biotechnological production than many of the "conventional" secondary metabolites.

Heinig and co-authors [14] may have resolved a longstanding mystery concerning the evolution of a complex terpenoid biosynthetic pathway in two distantly related organisms: They evaluated Taxol biosynthesis in Taxomyces andreanae (which should, fide Seifert et al. 2011, in future be regarded as a species of Cladorrhinum) and various other endophytic fungi derived from Taxus plants. Using a combination of state of the art methodology comprising analytical chemistry, molecular biology and genomics, they were unable to find any sound evidence that genes encoding for the biosynthesis of Taxol are present in the endophytic fungi. This anticancer compound was only detected in traces in primary cultures of the endophytes, but soon disappeared after several sub cultivation steps. These findings appear to explain why no sustainable process of taxol biosynthesis has yet been established, despite the fact that the compound had been detected in numerous previous studies since the first report of this "sensational" discovery in 1993. 
Any approach to obtain phytochemicals through biotechnological production of fungi should be analysed critically. Historical cases are apparent where important plant metabolites such as the gibberellin phytohormones were first isolated from a fungal overproducer, long before they could be detected in the plants. Such phenomena have been studied intensively, revealing interesting homologies and convergent evolutionary developments in distantly related organisms (cf. Bömke and Tudzynski 2009). On the other hand, there seems to be no lack of supply for Taxol derivatives, since the compound can be produced at the industrial scale either by harvesting Taxus needles in a sustainable manner, or even by cultivation of plant cells that actually possess the biosynthetic genes, and subsequent simple chemical derivatisation of the resulting baccatin precursor. Most established drugs of plant origin can also be easily obtained in up to ton scale from high production plant cell lines or cultivars after substantial efforts have been made to establish such production processes

An apparent outcome from this issue is the fact that endophytic fungi also harbour their own arsenal of bioactive secondary metabolites. This enormous diversity of silent secondary metabolite biosynthetic genes in fungi has only recently become evident through the increasing availability of genome sequence data and the development of straightforward corresponding bioinformatic tools and molecular genetic methods for their characterisation. Since most plants have been studied exhaustively for bioactive secondary metabolites, while only a small fraction of the fungal biodiversity has hitherto been even isolated into pure culture (let alone, studied extensively for biotechnological applications!), the chances to discover novel, non-generic chemical entities that are specifically produced by the fungi themselves are much higher (see reviews of Aly et al. 2010, 2011; Debbab et al. 2011, 2012). The phenomenon of horizontal gene transfer between endophytic fungi and their plant hosts and the study of the underlying molecular mechanisms, however, remain to be of great academic interest.

Hence, fungal endophytes are extremely attractive microorganisms for future studies in both basic and applied research. This special issue should further stimulate interdisciplinary international collaborations in this field, at European as well as at a global scale!
Acknowledgments This special issue was compiled within a period of 7 months. We would like to thank all authors for their timely submisssions and our fellow editors as well as numerous reviewers and the staff of the Editorial office, for helping to meet the deadlines. Support by COST Action FA1103 is gratefully acknowledged.

\section{References}

Aly AH, Debbab A, Kjer J, Proksch P (2010) Fungal endophytes from higher plants: a prolific source of phytochemicals and other bioactive natural products. Fungal Divers 41:1-16

Aly AH, Debbab A, Proksch P (2011) Fifty years of drug discovery from fungi. Fungal Divers 50:3-19

Bills GF, González-Menéndez V, Martín J, Platas G, Fournier J, Peršoh D, Stadler M (2012) Hypoxylon pulicicidum sp. nov. (Ascomycota, Xylariales), a pantropical Insecticide-producing endophyte. PLoS One 7(10):e46687. doi:10.1371/journal.pone.0046687

Bömke C, Tudzynski B (2009) Diversity, regulation and evolution of the gibberellin biosynthetic pathway in fungi compared to plants and bacteria. Phytochemistry 70:1876-1893

Debbab A, Aly AH, Proksch P (2011) Bioactive secondary metabolites from endophytes and associated marine derived fungi. Fungal Divers 49:1-12

Debbab A, Aly AH, Proksch P (2012) Endophytes and associated marine derived fungi-ecological and chemical perspectives. Fungal Divers 57:45-83

Huang WY, Cai YZ, Surveswaran S, Hyde KD, Corke H, Sun M (2009) Molecular phylogenetic identification of endophytic fungi isolated from three Artemisia species. Fungal Divers 36:69-88

Kesting JR, Olsen L, Staerk D, Tejesvi MV, Kini KR, Prakash HS, Jaroszewski JW (2011) Production of unusual dispiro metabolites in Pestalotiopsis virgatula endophyte cultures: HPLC-SPE-NMR, electronic circular dichroism, and time-dependent density functional computation study. J Nat Prod 74(10):2206-2215

Kusari S, Hertweck C, Spiteller M (2012) Chemical ecology of endophytic fungi: origins of secondary metabolites. Chem Biol 19:792-798

Maneerat W, Phakhodee W, Ritthiwigrom T, Cheenpracha S, Deachathai S, Laphookhieo S (2012) Phenylpropanoid derivatives from Clausena harmandiana fruits. Phytochem Lett 6:18-20

Peršoh D, Melcher M, Flessa F, Rambold G (2010) First fungal community analyses of endophytic ascomycetes associated with Viscum album ssp. austriacum and its host Pinus sylvestris. Fungal Biol 114:585-596

Seifert K, Morgan-Jones G, Gams W, Kendrick B (2011) The Genera of Hyphomycetes. CBS Biodiversity Series 9

Stadler M (2013) COST action FA1103: European scientists investigating endophytic microrganisms and fungi. IMA Fungus 3(2):50-51

Stadler M, Læssøe T, Fournier J, Decock C, Schmieschek B, Tichy HV, Persoh D (2013) A polyphasic taxonomy of Daldinia (Xylariaceae). Stud Mycol. doi:10.3114/sim0016 